\title{
Ecología urbana y planificación: una convergéncia ineludible
}

\author{
Urban ecology and planning: a necessary convergence
}

\author{
Fabio Angeoletto', Mark D. E. Fellowes", Liliana Essi"', Jeater W. M. C. Santos'v, \\ Juciane M. Johann`, Deleon da Silva Leandrov', Nathalia Moraes MendonçavıI
}

\section{Resumen}

La urbanización global y masiva de los territorios es el más importante fenómeno ecológico de la actualidad. Los estándares de crecimiento urbano son determinantes para la conservación de la diversidad biológica, y asimismo para el grado de calidad de vida aceptable de las poblaciones urbanas. Contrariamente a la percepción de los biólogos y de otros académicos, de las ciudades como ambientes estériles y opuestos a la naturaleza, el reciente desarrollo de la ecología urbana, ciencia necesariamente interdisciplinaria, nos demuestra que ciudades son ecosistemas heterotróficos que necesitan ser planeados para que sus impactos en la biosfera sean disminuidos, y que en paralelo su capacidad de sostener la biodiversidad sea incrementada.

Palabras-clave: Ecología Urbana; Ecosistemas Urbanos; Biodiversidad Urbana; Planificación Urbana

\section{Abstract}

The global and massive urbanization of territories is one of today's most important ecological phenomena. The standards used in urban growth are vital for the conservation of biological diversity, and also affects the provision of a reasonable quality of life for urban populations. Contrary to the perception held by many biologists and other academics, where cities are sterile environments and the opposite of natural, the recent development of urban ecology, a necessarily interdisciplinary science, shows that cities are heterotrophic ecosystems that need to be planned in a manner that reduces their impacts on the biosphere, and that in parallel increases their ability to sustain biodiversity.

Keywords: Urban Ecology; Urban Ecosystems; Urban Biodiversity; Urban Planning

\footnotetext{
Mestrado em Geografia da UFMT, Campus de Rondonópolis, MT, Brasil - fabio_angeoletto@yahoo.es

" Universidade de Reading, Reino Unido - m.fellowes@reading.ac.uk

II' Universidade Federal de Santa Maria, Santa Maria, RS - liliana.essi@ufsm.br

Iv jeater@ufmt.br

$\checkmark$ Mestrado em Geografia da UFMT, Campus de Rondonópolis, MT, Brasil - jucijohann@yahoo.com.br

v1 Mestrado em Geografia da UFMT, Campus de Rondonópolis, MT, Brasil - deleon_roo@hotmail.com

VII Mestrado em Geografia da UFMT, Campus de Rondonópolis, MT, Brasil - hauch29@gmail.com
} 


\section{Un panorama de la urbanización en el} mundo

Actualmente, más de la mitad de la humanidad vive en ciudades, por lo cual presenciamos una expansión sin precedentes de los paisajes urbanos. Por ejemplo, las ciudades ocupan aproximadamente el 10,2\% de los áreas costeros del planeta (DEANBORN y KARK, 2010). Este es indudablemente el más importante fenómeno ecológico humano de los siglos XX y XXI, con reflejos sobre la biosfera y los seres humanos que no están aun plenamente cuantificados y comprendidos.

En Brasil, el proceso de urbanización resulto en un desplazamiento de millones de personas desde las zonas rurales hacia las ciudades. En 1890 la población urbana de Brasil era cerca de $10 \%$ yen pocas décadas ese cuadro se invierte: hoy $83 \%$ de los brasileros viven en ciudades (ANGEOLETTO, 2012). En un primer ciclo, los emigrantes se han desplazado hacia las ciudades grandes. Desde a mediados de los años 1990, se inicia un según ciclo de urbanización en Brasil, con un aumento considerable del número de ciudades medias (aquellas con poblaciones entre 100.000 y 500.000 habitantes), de personas viviendo en ellas y del área ocupada por ellas. Se puede observar nítidamente una disminución del área ocupada por las metrópolis (de un $28,23 \%$ para un $16,23 \%$ del área total), mientras que el área ocupada por ciudades medias evoluciona, desde 1970 hasta 2000, de un 11,77\% para un $27,23 \%$ del área total ocupada por las ciudades brasileñas. El número de ciudades medias también crece considerablemente en ese periodo, pasando de 40, en 1970, a 194, en 2000 (CARVALHO 2003; ANGEOLETTO, 2012).

Actualmente hay 258 ciudades medias en Brasil, de un universo de 5570 municipios. En ellas viven cerca de $25 \%$ de la población brasileña. Además, esas ciudades siguen creciendo más rápidamente que las ciudades pequeñas y grandes (ANGEOLETTO et al. 2016). Las tendencias actuales de la urbanización brasileña reflejan un fenómeno global. Contrariamente a la creencia general, el grueso del aumento de la población urbana mundial ocurre en ciudades medianas y pequeñas, cuyas capacidades de planificación y ejecución suelen ser muy débiles (MCPHEARSON et al 2016). En el inicio del siglo XXI, de los 3,3 mil millones de urbanitas, el $52 \%$ vivían en ciudades con menos de 500 mil personas. Hasta 2025, esas ciudades van a absorber aproximadamente la mitad del crecimiento esperado de la población urbana global. Además, los pobres constituirán una gran parte del futuro crecimiento poblacional urbano mundial (DUNN et al. 2006).

\section{Las ciudades medias de Brasil tienen ba-} jos estándares de planificación y gestión

Angeoletto et al. (2016), respeto a la efectividad de la gestión ambiental urbana en las ciudades medias de Brasil, dividen esas ciudades en dos grupos principales. Las ciudades medias pobres. Son aquellas cuyas características más notables son un gran porcentaje de analfabetos, poblaciones jóvenes, trabajadores con bajos sueldos y pocas acciones municipales de gestión ambiental. Este grupo de ciudades pobres está íntegramente concentrado en las regiones norte / noreste de Brasil.

Por otro lado, en oposición a las ciudades pobres del norte y noreste de Brasil, en las ciudades ricas predominan poblaciones adultas, con bajas tasas de natalidad, y, además, más escolarizadas y con sueldos mayores. Por lo tanto, con estándares de consumo más sofisticados que aquellos presentes en las ciudades pobres. Los coches y otros vehículos ilustran bien las diferencias de consumo entre las ciudades medias pobres y ricas de Brasil. Las ciudades del sur y sudeste de Brasil tienen una relación vehículos por habitante muy similar al de las ciudades de los países del primer mundo. En la ciudad de Maringá (Paraná) por ejemplo, esa relación es de un vehículo privado para cada 2,08 habitantes, mientras que Cametá (Pará) tiene un vehículo para cada 55,1 habitantes.

La baja relación ciudadanos/vehículo en las ciudades medias más prósperas apuntadas por el estudio de Angeoletto et al. (2016) suponen un importante problema de salud pública, por la contaminación emitida por vehículos (y la plétora de otros impactos asociados a su uso). Además, esos datos sugieren claramente que son necesarias medidas para la reducción del papel del coche, preponderante no sólo en el transporte sino también en la conformación de la morfología de las ciudades de Brasil. En realidad, en mayor o menor grado, es este un dilema de todas las ciudades brasileñas, dado que hay una rápida y creciente expansión de la flota nacional, que se puede explicar por diversos factores, entre ellos, estabilización de la economía, carencia de planeamiento del uso del suelo urbano, adopción de un modelo de transporte que prioriza los desplazamientos en vehículos privados y una preocupante carencia de inversión en el transporte público de masas (VIANNA et al., 2004); además de incentivos gubernamentales a la compra de vehículos a través de la reducción del IPI (imposto sobre productos industrializados) sobre la venta de coches y motocicletas, como una respuesta a la crisis económica de 2008 (ANGEOLETTO, 2012).

El número medio de funcionarios de las secretarias municipales de medio ambiente (u órganos similares) es de 13 por grupo de 100.000 habitantes, siendo de esos, solamente dos con carrera universitaria. Aunque las ciudades medias ricas del sur/sureste de Brasil existen más acciones de gestión ambiental, tres categorías de fiscalización se destacan por la baja ocurrencia, cualquiera que sea el grupo de ciudades: la fiscalización sobre la contaminación atmosférica producida por vehículos, la fiscalización de gasolineras, y la fiscalización sobre el uso de fertilizantes y pesticidas en cultivos agrícolas. La escasez de funcionarios dedicados al medio ambiente, así como la relativa poquedad de equipos y acciones de gestión ambiental en las ciudades medias de Brasil, sugieren un cuadro de poca efectividad ante los problemas e impactos ambientales que necesitan ser corregidos o evitados en esas ciudades (ANGEOLETTO et al. 2016).

Muchos de los problemas ambientales urbanos resultan de administraciones ineficaces, y de la falta de planificación y políticas urbanas coherentes, más que 
del proceso de urbanización per se, y en las ciudades brasileñas la gestión y planificación todavía no han sido herramientas ambientalmente efectivas (ANGEOLETTO y MORENO, 2009). Queda claro que, si bien las cuestiones ambientales han alcanzado importancia en la sociedad civil, poca prioridad es dada a los problemas ambientales en el ámbito municipal, en Brasil. Ni siquiera hemos alcanzado, en las ciudades medias, los estándares de sostenibilidad blanda de ciudades de países desarrollados (es decir, sostenibilidad para unos pocos parámetros, como los relativos a la calidad del aire en los ecosistemas urbanos). Hay poco personal para la elaboración de políticas públicas ambientales que sean eficaces para contestar los inmensos desafíos medioambientales de las ciudades brasileñas (ANGEOLETTO et al., 2016).

\section{Ecología Urbana: Ciencia necesariamen- te interdisciplinaria}

Según Angeoletto (2012) y Camps-Calvet et al. (2015), la ecología urbana es una ciencia que integra las teorías y metodologías de las ciencias naturales y sociales para investigar estándares y procesos de los sistemas ecológicos urbanos. La intersección entre las ciencias naturales y sociales es el paradigma central de la ecología urbana. El estudio de ecosistemas urbanos es un campo relativamente reciente de la ecología. Hasta recientemente la tradición intelectual occidental no ha logrado integrar naturaleza y sociedad, resultando en una insuficiencia teórica, respecto a las interrelaciones entre ecología y ciudades. La corriente separación entre ecología y sociología ha obstruido una comprensión más amplia de los ecosistemas ecológicos urbanos (MCPHEARSON et al., 2016).

El ecólogo barcelonés Jaume Terradas (2001) caracteriza las ciudades como ecosistemas heterotróficos que se organizan aumentando la entropía alrededor del planeta. Distintamente de los ecosistemas autotróficos (esencialmente estructurados por cadenas alimentarias compuestas por organismos fotosintéticos que hacen la conversión de energía solar en energía química, que a su vez alimentan grupos de organismos heterótrofos), los ecosistemas heterotróficos dependen de áreas externas a ellos para la obtención de energía, alimentos, fibras y otros materiales, y para la deposición de los desechos y contaminantes.

Arroyos y arrecifes de ostras también son ejemplos de ecosistemas heterotróficos. Sin embargo, las ciudades difieren de ellos por tres diferencias principales: 1) un tecno-metabolismo mucho más intenso por unidad de área, hecho que exige un flujo más intenso de energía, parcialmente sustentada por combustibles fósiles; 2) una considerable necesidad de entrada de materiales, como metales, para la producción de bienes de consumo no necesariamente relacionado a la supervivencia humana; y 3) una salida mucho mayor y más contaminante de desechos y residuos (COLLINS et al., 2000).

Aunque ocupen un área ínfima de la biosfera (algo entre 1 e $5 \%$ de la parte terrestre del globo), las ciudades influencian toda la biosfera, a través de sus inmensos flujos de entrada y de salida. En resumen, ciudades son ecosistemas que poseen ambientes de entrada (terri- torio donde se recogen materias primas diversas) y de salida (puntos de la biosfera que reciben los residuos del metabolismo urbano) mucho más grandes de que otros ecosistemas heterotróficos. Por ello, se puede afirmar que ciudades y sus procesos ecológicos no están circunscriptos a limites administrativos, geográficos o políticos (MCPHEARSON et al., 2016).

Por razones históricas, la biología de la conservación ha dividido el mundo en hábitats prístinos y degradados. No obstante, hace falta un cambio de mentalidades: la biología de la conservación debe volverse a los hábitats donde viven los seres humanos, y producir conocimiento sobre como dividir esos hábitats antropogénicos con especies silvestres (FAETH et al., 2011). De hecho, aunque el porcentual de áreas protegidas este aumentando mundialmente desde 1990, el número de especies amenazadas sigue creciendo (PNUMA, 2011), hechos que ponen de relieve la urgencia del desarrollo de mecanismos adicionales de conservación biológica.

\section{Biodiversidad y servicios ambientales en ecosistemas urbanos}

Seguramente, la conversión de suelos relevantes, o bien desde el punto de vista social (suelos agrícolas) o bien desde el punto de vista ambiental (bosques, manglares y otros ecosistemas) es el impacto más deletéreo de la urbanización. Lambin y Meyfroidt (2011), calcularon un incremento de 516 millones de hectáreas de suelos prístinos o agrícolas convertidos en ciudades, hasta 2030. Respecto a la biodiversidad, la urbanización en general disminuye la riqueza de especies para la mayoría de las comunidades bióticas, a despecho del incremento de biomasa de pájaros y artrópodos. Hay una excepción notable a ese estándar: en las ciudades, la riqueza de especies de plantas tiende a aumentar. La riqueza (número) de especies vegetales en los ecosistemas urbanos es usualmente más elevada que aquella encontrada en zonas rurales, o incluso en fragmentos de bosques (LUBBE et al., 2010; FAETH et al., 2011; ANGEOLETTO, 2012; HAND et al., 2016). Es cierto, sin embargo, que en los ecosistemas urbanos predominan especies ruderales y exóticas, estas últimas presentes en estos ambientes por introducción intencional (por ejemplo, en la ornamentación de espacios públicos o ajardinamiento), o accidental (invasoras y naturalizadas). Gran parte de las plantas ornamentales cultivadas en los diversos lugares del mundo, por ejemplo, no es nativa de estas regiones (HEIDEN et al., 2006), siendo el cultivo de especies autóctonas aún muy limitado.

Entre los ecosistemas de la biosfera, las ciudades son únicas en la intensidad de su dominación por los humanos, y reflejan grupos de vegetación singulares, que resultan de una mezcla de factores económicos, sociales, ambientales y tecnológicos. Asimismo, recursos sociales críticos como poder político, renta, prestigio y conocimiento producen una nítida diferenciación social en las ciudades y estándares espaciales de esos recursos sociales también son factores de influencia considerables en la distribución de recursos biofísicos y naturales en 
los ecosistemas urbanos, como, por ejemplo, la vegetación (GALLUZZI et al., 2010; HOSTETLER et al., 2011; ANGEOLETTO et al., 2015).

Parece haber una tendencia de predominio de especies exóticas, animales y vegetales, mientras la urbanización avanza. Especies nativas y exóticas son combinadas en las ciudades de manera absolutamente singular, creando lo que autores como Dunn et al. (2006) y McPhearson et al. (2016) llaman de nuevos ecosistemas, con consecuencias a la biodiversidad que apenas empezamos a comprender. En esos sistemas ecológicos, el impacto de la vegetación exótica sobre los niveles tróficos más elevados permanece un debate en abierto. Algunos estudios apuntan un aumento significativo de pájaros y mariposas en jardines dominados por vegetación nativa, mientras que otros sugieren que la diversidad de invertebrados y vertebrados no está necesariamente correlacionada a especies nativas (GODDARD et al., 2010).

Muchos son los beneficios proporcionados por la flora urbana, principalmente por especies arbóreo-arbustivas. Los árboles urbanos mitigan las islas de calor, mientras sus hojas adsorben contaminantes atmosféricos y fijan $\mathrm{CO}_{2}$. Las personas están menos expuestas a la radiación ultravioleta en áreas urbanas de vegetación leñosa abundante. También se pueden mencionar la protección del suelo y de cuerpos de agua urbanos. Además, árboles son hábitats significativos a la diversidad biológica en las ciudades, y las manchas urbanas de vegetación (jardines de viviendas, plazas, parques y fragmentos de vegetación autóctone) pueden actuar como corredores ecológicos o peldaños conectando paisajes urbanos y forestales (DÍAZ et al., 2011; CAMPS-CALVET, 2015).

Varios estudios epidemiológicos han correlacionado positivamente una mayor longevidad al acceso a espacios verdes, pues áreas copiosamente vegetadas, incluso quintais, poseen mayor capacidad de disminuir la contaminación atmosférica y las islas de calor urbano. Las islas de calor aumentan la incidencia de infartos del miocardio, resultantes de la demanda excesiva del sistema cardiovascular, por la necesidad de sangre para los mecanismos de regulación de la temperatura corporal. Además, áreas verdes estimulan la práctica de ejercicios físicos, como caminadas y jardinería (TZOULAS et al., 2007).

Los beneficios económicos incluyen la valoración de viviendas cuyos barrios son bien vegetados, menores costes con calefacción y refrigeración y más facilidad para atracción de capitales, negocios y empleos. Los costes con energía eléctrica pueden disminuir hasta un $30 \%$ en las viviendas, en correlación con una cobertura arbórea más abundante alrededor (CAMPS-CALVET et al., 2015; HAND et al., 2016).

Ecosistemas urbanos con espacios verdes más extensos y de mejor calidad son más resilientes, atraen más diversidad biológica y presentan una mayor provisión de servicios ecosistémicos. Sin embargo, aunque el conocimiento sobre las múltiples funciones y beneficios de las áreas verdes urbanas esté bien desarrollado, él no está perfectamente integrado a los procesos de design, planificación y gestión. Además, metodologías para la evaluación de esas áreas que puedan efectivamente sos- tener la toma de decisiones son en general inexistentes (HAND et al., 2016).

La riqueza de especies vegetales en los ecosistemas urbanos es usualmente alta, pero mal distribuida entre los barrios, hecho que se configura en un fenómeno de injusticia ambiental, o sea, en una desigualdad en el acceso a los recursos ambientales, en la definición de Aleixo et al. (2016). Barrios de mayor status socio-económico normalmente presentan una mayor diversidad vegetal, o bien en sus espacios públicos, como parques y plazas (los cuales normalmente no existen en los barrios pobres), o bien en los jardines domésticos de las viviendas (los quintais, un uso del suelo común en las ciudades). (ANGEOLETTO, 2012; HAND et al., 2016)

Estudios comparativos de la diversidad vegetal entre barrios pobres y barrios de clase media alta son bastante ilustrativos respecto a esas desigualdades. Por ejemplo, en la ciudad de Maringá (Paraná, Brasil), en los quintais del barrio operário Jardim das Torres hay 165 especies vegetales, mientras que en el barrio Zona 02 la riqueza de espécies vegetales presentes en los quintais es de 381 especies (ANGEOLETTO et al. 2015). Se trata de un fenómeno corriente en las urbes brasileñas (ANGEOLETTO, 2012). También en otros países acentuadamente desiguales, como África del Sur, iniquidades en el acceso a la vegetación caracterizan las ciudades (LUBBE et al., 2010). La democratización del acceso a los beneficios de la flora urbana podría ser alcanzada, en las ciudades brasileñas, con plantíos en los patios de los barrios pobres. Estos plantíos tendrán un mayor éxito si se ven precedidos por acciones de educación ambiental y por la producción de plantones de árboles que atiendan al doble objetivo de incrementar la cobertura arbórea y recuperar esos paisajes para la biodiversidad, como proponen Duarte (2018) y Beltrame y Beltrame (2018).

\section{El rol de los cursos de posgrado brasileños} en la conservación de la biodiversidad urbana

Como suelen decir los chinos, es mejor ser bueno en tu propia casa que quemar incienso en un templo distante. Por su enorme influencia sobre la biosfera, la planificación del crecimiento de las ciudades se ha convertido en uno de los más importantes desafíos del siglo XXI (UNFPA, 2007). Sin embargo, está en curso un proceso de descentralización, lo cual resulta en un aumento de las responsabilidades, que se acumulan sobre las ciudades pequeñas y medias. Por ello será preciso encontrar nuevas maneras de equipar esas ciudades, para que planifiquen la futura expansión, utilicen sosteniblemente sus recursos y ofrezcan servicios esenciales. Una de esas maneras es la ejecución de proyectos de extensión que mezclen la capacidad técnica de los cursos de posgrado de las universidades con recursos humanos, políticos y financieros de los ayuntamientos (ANGEOLETTO, 2012).

Poblaciones urbanas dependen de un vasto abanico de servicios ecosistémicos, como la polinización, la regulación climática, y la absorción de carbono. Por otro lado, la manutención de esos servicios, o bien localmente, o bien 
regionalmente y globalmente, depende crecientemente de cómo se manifiestan los estándares de desarrollo de las ciudades (TZOULAS y JAMES, 2010). Usualmente, ciudades están ubicadas en ambientes-clave: próximas de ríos, estuarios, manglares y bosques. Por lo tanto, ciudades suelen desarrollarse en escenarios cruciales a la conservación biológica, hecho que pone de manifiesto la importancia de la planificación de urbes más amigables a la vida silvestre.

La planificación adecuada de los sistemas ecológicos urbanos es tan importante a la conservación de la biodiversidad cuanto es el establecimiento de áreas naturales legalmente protegidas (PNUMA, 2011), una afirmación que el espeluznante avance de la urbanización no planeada o escasamente planificada corrobora: mundialmente, el tamaño total de las áreas urbanas excede el tamaño total de las áreas legalmente destinadas a la conservación biológica, incluso en países poco poblados. En los Estados Unidos, por ejemplo, esta es la realidad desde la década de 1990 (DEARBORN y KALK, 2010).

Hay un argumento aún más tajante para la comprensión del carácter ecosistémico de las ciudades. La mayoría de los seres humanos vive hoy en ciudades, y dependen de una gestión ambiental adecuada para la manutención de una calidad de vida aceptable. La importancia social, ambiental y para la planificación y gestión de los estudios de ecología urbana, pone de manifiesto la urgencia de la formación de cuadros especializados a través de los cursos de posgrado de las universidades brasileñas (ANGEOLETTO y SANTOS, 2015). Efectivamente, en barrios ya establecidos y en el desarrollo de nuevas áreas de viviendas, la salud, calidad de vida y bien estar de los vecinos podrían ser beneficiadas por una mejor comprensión de los sistemas ecológicos urbanos, y en particular por una más amplia comprensión de la biodiversidad urbana, y de cómo conservarla y además incrementarla en las ciudades (FAETH et al., 2011; ANGEOLETTO et al., 2015).

Aproximadamente un $60 \%$ del crecimiento urbano global previsto para hasta 2030 aún está por ocurrir (SECRETARIAT, 2012), y por lo tanto, tenemos una oportunidad única, en Brasil y globalmente, de calificar investigadores en los cursos de posgrado, y habilitarlos para producir conocimientos que permitan el desarrollo de formas más inteligentes de urbanización. El establecimiento de un dialogo entre académicos y gestores es un reto fundamental en el proceso de planificación ambiental urbana, pues, usualmente, gobiernos locales tienen un conocimiento muy limitado de cómo mantener la biodiversidad en los ecosistemas urbanos (TZOULAS y JAMES, 2010; HAND et al., 2016).

Ciertamente, comprender la inmensa complejidad de la ecología de las ciudades, ecosistemas influenciados no solo por factores ambientales, sino también por dinámicas sociales, económicas, políticas y culturales requiere el continuo desarrollo de metodologías interdisciplinarias. El número de personal investigador brasileño calificado para desarrollar estudios en ecología urbana (biólogos, arquitectos, geógrafos, sociólogos y otros) es claramente insuficiente (ANGEOLETTO, 2012). Cerca de 90\% de los estudios de ecología urbana son realizados en ciudades de países desarrollados (SECRETARIAT, 2012). Efectivamente, hay pocos estudios sobre la ecología de las ciudades brasileñas, en un momento histórico que, como hemos afirmado, la influencia de las ciudades sobre la biosfera se ha ampliado a una magnitud tal, que es imposible analizar separadamente las cuestiones ambientales y urbanas. El desafío de la conservación de la biodiversidad e de sus servicios ambientales (jimprescindibles a los seres humanos!) solo será superado a través del desarrollo de políticas urbanas más amigables a la fauna y flora, que, es importante resaltar, todavía no han sido creadas para las ciudades de Brasil (ANGEOLETTO y SANTOS, 2015).

Desde un punto de vista jurídico, la Ley brasileña está repleta de principios legales reclamando una planificación ambientalmente sostenible, principalmente para satisfacer las medianas y grandes ciudades. En este sentido, la Constitución Federal de Brasil de 1988 garantiza a todos los brasileños y extranjeros residentes en el país derecho a un medio ambiente ecológicamente equilibrado e impone el gobierno y la sociedad el deber de defenderlo y la conservó incluso para las generaciones futuras, reconociendo como titular este derecho que aún no ha sido diseñado, ya sea brasileño o no (art. 225). Además la Constitución establece una política de desarrollo urbano destinado a organizar la plena expresión de las funciones sociales de la ciudad y a garantizar el bienestar de sus habitantes (art. 182).

Municipios brasileños con poblaciones de 20.000 vecinos o más necesitan tener leyes específicas que regulen su política de desarrollo y la expansión urbana, la cual debe incluir espacios para la protección de la biodiversidad. En el estado brasileño de Mato Grosso, por ejemplo, se exige por ley que al menos el $10 \%$ de la superficie de la mancha urbana de las ciudades sean zonas verdes. Otros instrumentos guardan relación con la forestación de las vías públicas, protección de manantiales y áreas ribereñas, etc.

Desafortunadamente, estos instrumentos son todavía pocos usados en el proceso de planificación, entre otros motivos, porque la ecología urbana no ha sido todavía incorporada a los estándares de planificación y gestión urbanas (TERRADAS, 2001; ANGEOLETTO, 2012). Esos datos y argumentos demuestran, de manera cristalina la urgente necesidad del surgimiento de cursos de posgrado en ecología urbana, o por lo menos de líneas de investigación en ecología urbana en posgrados de ecología, arquitectura y urbanismo, geografía y ciencias ambientales. Las universidades brasileñas necesitan formar profesionales capacitados a lidiar con los inúmeros desafíos ambientales originados por la urbanización.

6 El futuro de las ciudades brasileñas: ¿Blade Runner o hotspots verdes?

Roger Waters compuso, en 1969, con sus compañeros de la banda de rock Pink Floyd la canción Cirrus Minor. La letra nos habla de disfrutes biofílicos, en un clima pastoril, mientras la melodía se mezcla al canto de un ruiseñor. Pero no se trata de nostalgia por una naturaleza que se perdió: 
In a churchyard by a river

Lazing in the haze of midday,

Laughing in the grasses and the graze.

Yellow bird, you are not alone

In singing and in flying on,

In laughing and in leaving.

Willow weeping in the water

Waving to the river daughters,

Swaying in the ripples and the reeds.

On a trip to Cirrus Minor,

Saw a crater in the sun

A thousand miles of moonlight later.

La placida contemplación de elementos naturales tan caros a los humanos, desde un patio, no es una escena del siglo XVIII. Pronto el poeta nos habla de un viaje espacial, situándonos en el futuro. Un futuro seductor, urbano, donde miríadas de criaturas vivas, tan llenas de colores, conviven con una profusión de avances tecnológicos que nos permiten realizar todos los sueños engendrados por nuestras cabezas primates. ¿Qué futuro urbano tendremos? ¿Megalópolis grises como las representadas en la película cult Blade Runner? Ciertamente se multiplicarán ciudades, las cuales serán pesadillas de concreto y humo o puntos calientes de diversidad biológica. ¿Habrá una efectiva agenda para las cuestiones medioambientales urbanas? ¿Habrá capacidad técnica para un crecimiento urbano más elegante y más inteligente, en Brasil?

\section{Agradecimientos}

CAPES y Ministério Público do Estado de Mato Grosso

\section{Referéncias}

Aleixo B et al. Human Right in Perspective: Inequalities in Access to Water in a Rural Community of the Brazilian Northeast. Ambiente \& Sociedade. 2016; 19(1): 63-84. 2016.

Angeoletto F. Planeta Ciudad: ecología urbana y planificación de ciudades medias de Brasil. Tesis doctoral. Doctorado en Ecología de la Universidad Autónoma de Madrid. 2012.

Angeoletto F, Moreno M. Tendencias Socio-ambientales de Ciudades Brasileñas. In: Mendes CM, Töws RL (org.). Geografia Urbana e Temas Transversais. Editora da Universidade Estadual de Maringa. Maringa, Brasil, 2009.

Angeoletto F, Santos JWMC. Los biólogos brasileños no habitan en el planeta ciudad: por qué es urgente formar ecólogos urbanos. Revista Espaço Acadêmico. 2015; 14(165): 74-82.

Angeoletto F, Santos JWMC, Sanz JPR. ¿Hay flores en el jardín? La vegetación cultivada en patios urbanos a través de un gradiente social. Paisagem e Ambiente. 2015; (35): 119-135.
Angeoletto F. et al. Tipología Socio-Ambiental de las Ciudades Medias de Brasil: Aportes para un Desarrollo Urbano Sostenible. Urbe. Revista Brasileira de Gestão Urbana. 2016; 8(20): 272-287.

Beltrame, T. F., \& Beltrame, A. (2018). Um relato de recuperação de uma área: conscientização por meio da educação ambiental. Revista Ciência em Extensão, 14(1), 141-153.

Camps-Calvet $\mathrm{M}$ et al. Ecosystem services provided by urban gardens in Barcelona, Spain: Insights for policy and planning. Environmental Science \& Policy, 2016.

Carvalho E. Exclusão social e crescimento das cidades medias brasileiras. Scripta Nova - Revista Electrônica de Geografía y Ciencias Sociales. 2003; 6(146).

Collins JP, Kinzig A, Grimm NB et al. A new urban ecology: Modeling human communities as integral parts of ecosystems poses special problems for the development and testing of ecological theory. American. Scientist. 2000; 88: 416-425.

Dearborn DC, Kark S. Motivations for conserving urban biodiversity. Conservation Biology. 2010; 24(2): 432-440.

Díaz F, Quetier F, Caceres DM et al. Linking Functional Diversity and Social Strategies in a Framework for Interdisciplinary Analysis of Nature's Benefits to Society. Proceedings of the National Academy of Sciences. 2011; 108(3) 895-902.

Duarte, M. B. D. C. P., dos Santos, M. F. P., de Melo Falcão, N. A., \& dos Santos, A. C. M. (2018). O trabalho de campo na recuperação da mata ciliar do Riacho Gulandim. Anais do I Colóquio Internacional de Educação Geográfica e do IV Seminário Ensinar Geografia na Contemporaneidade, 1(1), 345-356.

Dunn RR et al. The pigeon paradox: dependence of global conservation on urban nature. Conservation Biology. 2006; 20(6): 1814-1816.

Faeth SH, Bang C, Saari S. Urban biodiversity: patterns and mechanisms. Annals of the New York Academy of Sciences. 2011; 1223(1): 69-81.

Galluzzi G, Eyzaguirre P, Negri V. Home gardens: neglected hotspots of agro-biodiversity and cultural diversity. Biodiversity and Conservation. 2010; 19(13): 3635-3654.

Goddard MA, Dougill AJ, Benton TG. Scaling up from gardens: biodiversity conservation in urban environments. Trends in Ecology and Evolution. 2010; 25: 90-98.

Hand KL et al. A novel method for fine-scale biodiversity assessment and prediction across diverse urban landscapes reveals social deprivation-related inequalities in private, not public spaces. Landscape and Urban Planning. 2016; 151:33-44. 
Heiden, G. et al. Considerações sobre o uso de plantas ornamentais nativas. Revista Brasileira de Horticultura Ornamental. 2006; 12(1): 2-7.

Hostetler M, Allen W, Meurk C. Conserving urban biodiversity? Creating green infrastructure is only the first step. Landscape and Urban Planning. 2011; 100(4): 369-371.

Lambin EF, Meyfroidt P. Global land use change, economic globalization, and the looming land scarcity. Proceedings of the National Academy of Sciences. 2011; 108(9): 3465-3472.

Lubbe CS et al. Political legacy of South Africa affects the plant diversity patterns of urban domestic gardens along a socio-economic gradient. Scientific Research and Essays. 2010; 5(19): 2900-2910.

Mcphearson T et al. Advancing Urban Ecology toward a Science of Cities. BioScience. 2016; 16: 113-124.

Secretariat of the Convention on Biological Diversity. Cities and Biodiversity Outlook. Montreal, 64 p. 2012.

PNUMA (Programa de las Naciones Unidas para el Medio Ambiente). Anuario PNUMA - Temas Emergentes en Nuestro Medio Ambiente Global. PNUMA. Nairobi, Kenya. 2011.

Tzoulas K et al. Promoting ecosystem and human health in urban areas using Green Infrastructure: A literature review. Landscape and Urban Planning. 2007; 81: 167-178.

Tzoulas K, James P. Making biodiversity measures accessible to nonspecialists: an innovative method for rapid assessment of urban biodiversity. Urban Ecosystems. 2010; 13: 113-127.

Terradas J. Ecología Urbana. Editorial Rubes. Barcelona, Espana. 2001.

UNFPA. Estado de la Población Mundial 2007. United Nations Population Fund. New York, USA. 2007.

Vianna $\mathrm{M}$ et al. Intelligent transportation systems and parking management: implementation potential in a Brazilian city. Cities. 2004; 21(2): 137-148. 